\title{
Review: antibiotics reduce the duration and severity of travellers' diarrhoea
}

De Bruyn G, Hahn S, Borwick A. Antibiotic treatment for travellers'diarrhoea. Cochrane Database Syst Rev. 2000;(3):CD002242 (latest version 21 May 2000).

Source of funding: Department for International Development UK

\section{QUESTION: In patients with travellers' diarrhoea, are antibiotics more effective and safer than placebo or another antibiotic in resolving diarrhoea?}

\section{Data sources}

Studies were identified by searching the Cochrane Infectious Diseases Group trials register, Medline (from 1966), EMBASE/Excerpta Medica (from 1988), abstracts of conferences and meetings, and bibliographies of relevant studies and by contacting experts in the field.

\section{Study selection}

Studies were selected if they were randomised controlled trials in any language, they involved adults or children $\geqslant 5$ years of age who were travelling outside their usual country of residence for $<6$ months and had non-bloody diarrhoea lasting $\leqslant 14$ days, and any antibiotic was compared with placebo or another antibiotic

\section{Data extraction}

Data were extracted independently by 2 reviewers on study quality (allocation concealment, generation of allocation sequence, and inclusion of all randomly allocated patients) and outcome measures (duration of diarrhoea [time to last unformed stool], severity [number of loose stools passed per $24 \mathrm{~h}$ period], and tolerability).

\section{Main results}

20 trials were included. 12 trials were placebo controlled (1474 patients). 10 of the placebo controlled trials reported the primary outcome of time to last unformed stool. The data could not be pooled, but all 10 trials reported a shorter duration of diarrhoea that was statistically significant among patients who received antibiotics (except in one group of patients who received ofloxacin) than among those who received placebo. In 6 trials reporting the number of patients cured of diarrhoea by 72 hours, antibiotics were more effective than placebo (table). 2 trials that reported on severity showed antibiotics to be more effective than placebo in relieving severity for each 24 hour period up to 72 hours: patients receiving antibiotics had a mean of 1.6 (95\% CI 0.5 to 2.7 ) fewer unformed stools from 0 to 24 hours, 2.1 (CI 1.4 to 2.8) fewer from 25 to 48 hours, and 1.4 (CI 0.8 to 1.9 ) fewer from 49 to 72 hours. 5 trials reporting adverse effects showed that more side effects were reported by patients receiving antibiotics than by those receiving placebo (odds ratio 2.37 , CI 1.5 to 3.8 ).

\section{Conclusion}

In patients with travellers' diarrhoea, antibiotics reduce the duration and severity of diarrhoea better than placebo but are associated with increased side effects.

For correspondence: Dr G de Bruyn, Department of Medicine, Baylor College of Medicine, Fondren-Brown Building, 6565 Fannin Street, Houston, TX 77030 , USA. Fax +1 7137931522

Antibiotics v placebo for travellers' diarrhoea (6 trials)*

\begin{tabular}{lllll} 
& \multicolumn{2}{c}{ Weighted event rates } & \\
\cline { 2 - 3 } Outcome & Antibiotics & Placebo & RBI (95\% CI) & NNT (CI) \\
\cline { 1 - 2 } Patients cured at 72 hours & $84 \%$ & $50 \%$ & $68 \%(49$ to 89$)$ & 3 (3 to 4$)$ \\
\hline
\end{tabular}

\section{COMMENTARY}

De Bruyn et al have done an excellent job with this meta-analysis given the limited information available from clinical trials. No doubt exists that duration of diarrhoea can be statistically significantly shortened with the use of antibiotics. The main question that arises from these investigations, however, is the clinical significance of this finding. The primary outcome of how much shorter the time to last unformed stool could not be determined. The authors were clearly frustrated with this outcome, as they state in their own conclusions. Does this review help us in treating travellers with diarrhoea? Options for treatment are antibiotic prophylaxis, antimotility agents, treatment with antibiotics once disease develops, or no drug treatment but treatment with rehydration as required.

The disease itself is difficult to typify because $25 \%$ to $50 \%$ of cases have no specific aetiological agent identified. ${ }^{1}$ The diarrhoeal component lasts for 1 to 5 days, depending on the aetiological agent, and is usually self limited. Prevention strategies aimed at dietary controls have also been controversial because of poor compliance and uncertain effectiveness. ${ }^{2}$ Prophylaxis with bismuth preparations and antibiotics has been effective, but it must be taken continuously while travelling and may be more expensive than symptomatic treatment alone. Patients who might benefit from this strategy are those who have the most to lose if they become sick, such as immunocompromised people or executive travellers in a short term, high risk exposure situation.

The bottom line is that travellers' diarrhoea is a self limiting disorder that responds well to symptomatic treatment. Whether this treatment should be antibiotics or antimotility agents alone is not known. Furthermore, as this study shows, the clinical benefit in terms of time with symptoms is not clear. The current recommendation that travellers undergo pre-travel counselling and carry medications for symptomatic treatment is not changed by this information, but the need for further study is aptly illustrated.

Neil E Gibson, MD, MSc University of Alberta Edmonton, Alberta, Canada

1 Ericsson CD. Travelers' diarrhea. Epidemiology, prevention, and self-treatment. Infect Dis Clin North Am 1998;12:285-303.

2 Steffen R, van der Linde F, Gyr K, et al. Epidemiology of diarrhea in travelers. JAMA 1983;249:1176-80
*Abbreviations defined in glossary; RBI, NNT, and $\mathrm{Cl}$ calculated from data in article. 